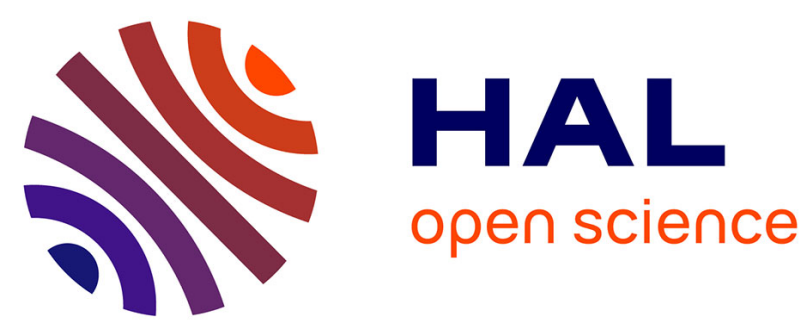

\title{
Comparative assessment of knee joint models used in multi-body kinematics optimisation for soft tissue artefact compensation.
}

\author{
Vincent Richard, Aurelio Cappozzo, Raphaël Dumas
}

\section{To cite this version:}

Vincent Richard, Aurelio Cappozzo, Raphaël Dumas. Comparative assessment of knee joint models used in multi-body kinematics optimisation for soft tissue artefact compensation.. Journal of Biomechanics, 2017, 62, pp.95-101. 10.1016/j.jbiomech.2017.01.030 . hal-01627863

\section{HAL Id: hal-01627863 \\ https://hal.science/hal-01627863}

Submitted on 2 Nov 2017

HAL is a multi-disciplinary open access archive for the deposit and dissemination of scientific research documents, whether they are published or not. The documents may come from teaching and research institutions in France or abroad, or from public or private research centers.
L'archive ouverte pluridisciplinaire $\mathbf{H A L}$, est destinée au dépôt et à la diffusion de documents scientifiques de niveau recherche, publiés ou non, émanant des établissements d'enseignement et de recherche français ou étrangers, des laboratoires publics ou privés. 


\section{Accepted Manuscript}

Comparative assessment of knee joint models used in multi-body optimisation for soft tissue artefact compensation

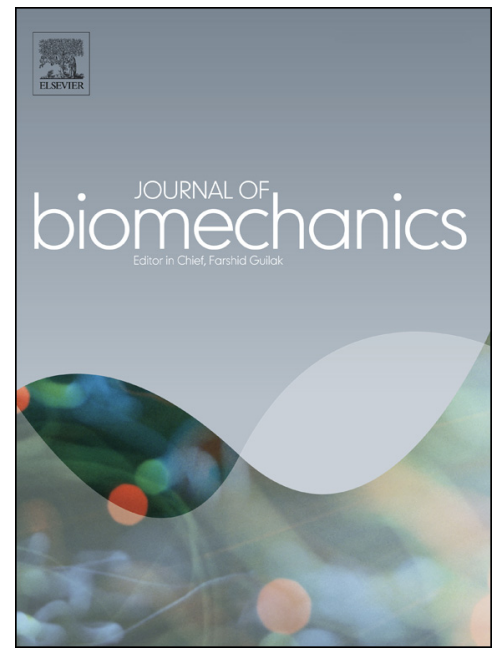

To appear in: $\quad$ Journal of Biomechanics

Accepted Date: $\quad 16$ January 2017

Please cite this article as: V. Richard, A. Cappozzo, R. Dumas, Comparative assessment of knee joint models used in multi-body optimisation for soft tissue artefact compensation, Journal of Biomechanics (2017), doi: http:// dx.doi.org/10.1016/j.jbiomech.2017.01.030

This is a PDF file of an unedited manuscript that has been accepted for publication. As a service to our customers we are providing this early version of the manuscript. The manuscript will undergo copyediting, typesetting, and review of the resulting proof before it is published in its final form. Please note that during the production process errors may be discovered which could affect the content, and all legal disclaimers that apply to the journal pertain. 
Comparative assessment of knee joint models used in multi-body optimisation for soft tissue artefact compensation

Vincent Richard $^{1,2,3}$, Aurelio Cappozzo ${ }^{2,3}$, Raphaël Dumas ${ }^{1,3}$

${ }^{1}$ Univ Lyon, Université Claude Bernard Lyon 1, IFSTTAR, UMR_T9406, LBMC, F69622, Lyon, France

${ }^{2}$ Università degli Studi di Roma - Foro Italico, Department of Movement, Human, and Health Sciences, Rome, Italy

${ }^{3}$ Interuniversity Centre of Bioengineering of the Human Neuromusculoskeletal System, Università degli Studi di Roma "Foro Italico", Rome, Italy

Wordcount:

Abstract: 211 words

Main text: 3454 words 


\subsection{Abstract}

Estimating joint kinematics from skin-marker trajectories recorded using stereophotogrammetry is complicated by soft tissue artefact (STA), an inexorable source of error. One solution is to use a bone pose estimator based on multi-body optimisation (MBO) embedding joint constraints to compensate for STA. However, there is some debate over the effectiveness of this method. The present study aimed to quantitatively assess the degree of agreement between reference (i.e., artefact-free) knee joint kinematics and the same kinematics estimated using MBO embedding six different knee joint models. The following motor tasks were assessed: level walking, hopping, cutting, running, sit-to-stand, and step-up. Reference knee kinematics was taken from pin-marker or biplane fluoroscopic data acquired concurrently with skin-marker data, made available by the respective authors. For each motor task, Bland-Altman analysis revealed that the performance of $\mathrm{MBO}$ varied according to the joint model used, with a wide discrepancy in results across degrees of freedom (DoFs), models and motor tasks (with a bias between $-10.2^{\circ}$ and $13.2^{\circ}$ and between $-10.2 \mathrm{~mm}$ and 7.2 $\mathrm{mm}$, and with a confidence interval up to $\pm 14.8^{\circ}$ and $\pm 11.1 \mathrm{~mm}$, for rotation and displacement, respectively). It can be concluded that, while MBO might occasionally improve kinematics estimation, as implemented to date it does not represent a reliable solution to the STA issue.

\section{Keywords}

Multi-body optimisation; kinematic constraints; soft tissue artefact; degree of agreement; knee joint; human locomotion

\section{Introduction}

Joint kinematics estimation commonly relies on methods involving a mechanical model of the locomotor apparatus together with the stereophotogrammetric tracking of skin-marker 
trajectories. However, soft tissue artefact (STA), i.e., the relative movement between the skinmarkers and the underlying bones, introduces errors that jeopardise the information content of the skeletal motion estimation (Leardini et al., 2005; Peters et al., 2010). Since the artefact has a frequency content similar to that of bone movement, the problem cannot be solved by filtering (Chiari et al., 2005).

Multi-body optimisation (MBO) is increasingly used with the intent to compensate for STAs. The method embeds a rigid multi-body system and kinematic models of the joints involved, which means that the degrees of freedom (DoFs) of the joints are constrained (Andersen et al., 2009; Bonnechère et al., 2015; Charlton et al., 2004; Duprey et al., 2010; Lu and O'Connor, 1999; Ojeda et al., 2014; Reinbolt et al., 2005).

Various mechanical linkages representing the knee joint and embedded in MBO have been described. These involve major simplifications with respect to real and subject-specific joints, and have less than six independent DoFs. The hinge joint (Andersen et al., 2009; Reinbolt et al., 2005) allows rotation about only the flexion-extension axis. The spherical joint, the most common representation of the knee in MBO (Charlton et al., 2004; Lu and O'Connor, 1999; Ojeda et al., 2014), allows all rotational movements but no translation. These models provide, in most cases, a rather inadequate 3D representation of the physiological movement of the knee (Andersen et al., 2010; Clément et al., 2017). Parallel mechanisms have also been used (Duprey et al., 2010; Gasparutto et al., 2015; Valente et al., 2015), the principle of which relies on compound joints representing an assembly of simple mechanical linkages. Although these models generally allow most rotations and translations, they couple the DoFs thereby prescribing displacements in a deterministic way (i.e., kinematics is imposed by the geometry of the model). A different approach consists in representing the behaviour of the knee directly by mathematically coupling the DoFs (Bonnechère et al., 2015; Li et al., 2012; Scheys et al., 2011), with up to five DoFs driven by the flexion angle. A more recent modelling approach 
relies on a knee joint stiffness matrix and minimization of the relevant deformation energy (Richard et al., 2016).

Based on a number of studies assessing $\mathrm{MBO}$, it may be concluded that no fully satisfactory knee joint model has been found yet (Andersen et al., 2010; Clément et al., 2017; Gasparutto et al., 2015; Richard et al., 2016). However, each of these assessment studies was performed on a single motor task (i.e., level walking, stepping-up, running or squatting). Moreover, some motor tasks (e.g., hopping, cutting) have not yet been investigated. Nor have all the abovementioned joint models been compared to date. Finally, existing comparisons have been based on the root mean square error between estimated and reference kinematics, without performing any deeper analysis regarding the relevant degree of agreement (McLaughlin, 2013).

This study aimed to comprehensively compare the performance of MBO embedding six different knee joint models selected from those proposed in the literature. This was made possible thanks to the availability of concurrently acquired reference, virtually artefact-free, bone kinematics and skin-marker data (Cereatti et al., 2016). The following motor tasks performed by able-bodied volunteers were analysed: level walking, hopping, cutting, running, sit-to-stand, and step-up. The degree of agreement between the reference and the MBOestimated joint kinematics was assessed by Bland-Altman analysis as well as using the relevant root mean square error and determination coefficient.

\section{Materials and Methods}

\subsection{MBO framework}

In this study, each bony segment is fully located and oriented (i.e., bone pose) in the global reference coordinate system by means of natural coordinates (de Jalon et al., 1994; Dumas 
and Chèze, 2007). Only the knee joint (i.e., the tibio-femoral joint) was considered in the study, meaning that the only segments involved in the MBO were the shank and the thigh.

Three types of constraints are typically used in MBO: driving constraints $\boldsymbol{\Phi}^{m}$, rigid body constraints $\boldsymbol{\Phi}^{r}$, and kinematic constraints $\boldsymbol{\Phi}^{k}$. The constraints are split into two sets of equations. A set of "soft" constraints contains the equations that may be violated (i.e., $\boldsymbol{\Phi}^{m}$ ). These constraints define the objective function of MBO, $f=\left(\boldsymbol{\Phi}^{m}\right)^{T} \boldsymbol{\Phi}^{m}$. A set of "hard" constraints contains the equations that must be fulfilled $\left(\boldsymbol{\Phi}^{r}, \boldsymbol{\Phi}^{k}\right)$. In this framework, MBO is thus, to be regarded as a constrained optimisation problem. Note that a subset of the kinematic constraints $\boldsymbol{\Phi}^{k_{1}}$, especially in the case of ligaments, may be considered as "soft" constraints and appended in the objective function, $f=\left(\begin{array}{c}\boldsymbol{\Phi}^{m} \\ \boldsymbol{\Phi}^{k_{1}}\end{array}\right)^{T}[\mathbf{W}]\left(\begin{array}{l}\boldsymbol{\Phi}^{m} \\ \boldsymbol{\Phi}^{k_{1}}\end{array}\right)$ (with a weight matrix W).

The present study considered the following knee joint models described in the literature and implemented, using natural coordinates, as kinematic constraints within MBO:

- None: no joint model, where the relative movement of the tibia and the femur are independent from each other and joint dislocation is therefore possible (this is, of course, a borderline case of $\mathrm{MBO}$ );

Spherical: spherical joint model, allowing the three rotations while impeding the three displacements;

- Hinge: hinge joint model, allowing only one rotation about the flexion-extension axis while impeding the other DoFs;

- Parallel: parallel mechanism with minimized ligament length variation, where two sphere-on-plane contacts stand for the contact between femoral condyles and tibial plateau. 
- Coupling: coupling curves between the DoFs, where internal rotation and adduction angles, as well as anterior and proximal displacements are functions of the extension angle through polynomial functions, and where lateral displacement is impeded;

- Elastic: elastic joint model based on the stiffness matrix, where all six DoFs are defined by the minimisation of the deformation energy.

A detailed description of the MBO method embedding the different models (i.e., kinematic constraints $\boldsymbol{\Phi}^{k}$ ) can be found in Duprey et al. (2010), Gasparutto et al. (2015) and Richard et al. (2016). More specifically, for model Parallel, the model geometry was taken from ParentiCastelli and Sancisi (2013) and, for model Coupling, the coupling curves between the DoFs were an adaptation, due to a different sign convention, of those provided by Walker et al. (1988). Note that the MBO embedding model None is actually equivalent to a single-body optimisation (e.g., Soderkvist and Wedin, 1993).

\subsection{Joint kinematics estimation}

Joint coordinate systems used to compute the kinematics of the knee joint were defined so as to satisfy the conventions for axes and Euler sequence proposed by the ISB (Wu et al., 2002). The actual joint angles and displacements (extension, adduction, and internal rotation angles, and lateral, anterior, and proximal displacements) were computed from the natural coordinates (Dumas et al., 2012).

\subsection{Experimental data}

Right thigh and shank movement data from a single trial for each of the selected motor tasks, performed by able-bodied male subjects, were used for the analysis. These data were obtained from the datasets reported in Cereatti et al. (2016). They included both virtually artefact-free bone-pose data, obtained using either pin-markers or biplane fluoroscopy, and concurrently acquired skin-marker data. The bony segment coordinate systems were defined based on bone 
anatomy and the reference positions of the skin-markers with respect to these coordinate systems were defined as their mean positions over the duration of the motor task. The data for level walking, hopping and cutting were from one volunteer (age: 22 years, mass: $63 \mathrm{~kg}$, height: $1.75 \mathrm{~m}$; Benoit et al., 2006), while the data for running were from another volunteer whose anthropometric features were unknown (Reinschmidt et al., 1997). Relevant artefactfree data were obtained using pins inserted in the distal femur and proximal tibia. Data for the step-up and sit-to-stand tasks were from one male volunteer (age: unknown, mass: $83 \mathrm{~kg}$, height: $1.75 \mathrm{~m}$ ) and artefact-free data were obtained via biplane fluoroscopy (Tsai et al., 2011). Further details concerning the experimental set-ups, the definition of bony segment coordinate systems and relevant calibration and registration procedures used for the different datasets can be found in Cereatti et al. (2016) and in the above-mentioned references.

\subsection{Assessment}

For each of the six motor tasks, reference (i.e., artefact-free) femur and tibia pose and knee joint angles and displacements were reconstructed using pin-marker or biplane fluoroscopy data. Femur and tibia pose and knee joint kinematics were also estimated using the concurrently acquired skin-marker data and six MBO procedures each embedding one of the above-illustrated knee joint models (None, Spherical, Hinge, Parallel, Coupling and Elastic). The degree of agreement between the joint angles and displacements derived from the six $\mathrm{MBO}$ procedures and the reference kinematics was assessed through Bland-Altman analysis (Bland and Altman, 1986). The bias (b) and confidence interval (CI; i.e., 1.96 standard deviation) were calculated. The root mean square error (RMSE) and coefficient of determination $\left(R^{2}\right)$ were also calculated for the sake of comparison with previous studies. Note that, when using the models Spherical and Hinge, displacements were null, thus the relevant coefficient of determination could not be computed. 


\section{Results}

Full results are presented here for three motor tasks: level walking, hopping and cutting, while results for the other motor tasks are reported in Supplementary Material. Note that the results reported in the body of the paper and those in Supplementary Material lead to the same general conclusions.

\subsection{Kinematics}

Both reference joint angles and displacements and those estimated using MBO embedding the six joint models, are represented in Figure 1, Figure 2, and Figure 3 for level walking, hopping, and cutting, respectively. Overall, the extension angles estimated through the six MBO procedures and the reference angles exhibited similar patterns. However, conflicting results emerged with regard to the other DoFs. Depending on the motor task and the DoF considered, large discrepancies were observed in the kinematic outcomes of the MBO for any given joint model. Note that the characteristics of the knee models meant that model Spherical provided null displacements, model Hinge provided null displacements as well as null adduction and internal rotation angles, and model Coupling provided null lateral displacement. No general tendency could be extracted from the overall analysis of the joint angle and displacement time histories. Nonetheless, the kinematics obtained using MBO embedding model None (i.e., no constraints) did not stand out in any way from the other five models.

FIGURES 1, 2 AND 3 ABOUT HERE 


\subsection{Statistics}

The statistical parameters are reported in Table 1, Table 2 and Table 3 for level walking, cutting, and hopping, respectively. In most cases, the lowest bias $b$ was obtained using MBO with model None, particularly for the joint angles. However, $C I$ was higher than that obtained with the other models. As observed from the joint angle and displacement time histories, the different models embedded in the MBO provided disparate results. There were marked differences in the RMSE and $R^{2}$ values obtained from the different joint models, motor tasks and DoFs.

For level walking, models Hinge and Coupling provided significantly higher $b$ values for joint angles, with $7.9^{\circ}$ and $7.2^{\circ}$ respectively for internal rotation, while the lowest $b$ value was provided by model None, with an absolute value between $0.0^{\circ}$ and $0.2^{\circ}$. MBO embedding models Spherical, Hinge, Parallel, Coupling and Elastic generally provided lower CI than MBO embedding model None, except for extension. Models Parallel and Elastic provided the highest $b$ values overall for displacements, with absolute values of up to $5 \mathrm{~mm}$ obtained for lateral displacement with model Parallel and for anterior displacement with model Elastic. For cutting, the lowest CI was provided by model Hinge for extension, by model Elastic for adduction, internal rotation and lateral displacement, and by model Coupling for anterior and proximal displacements, while for $b$ the lowest values were obtained with model Elastic for extension, with model None for adduction, with model Spherical for internal rotation, and with models Spherical and Hinge for all displacements. For hopping, $b$ was found null for extension with models None, Spherical and Elastic and for proximal displacement with Spherical, Hinge and Parallel. The lowest $b$ values were obtained with models None and Elastic for adduction, with model None for internal rotation and lateral displacement, and with model Coupling for anterior displacement, while model None provided the highest $C I$ values for adduction, internal rotation, lateral and anterior and the lowest for extension. 


\section{TABLES 1, 2 AND 3 ABOUT HERE}

\section{Discussion}

The aim of this study was to evaluate how effective MBO is in limiting the propagation of STA to joint kinematics estimates when various knee joint models are used. The joint models assessed in this study were selected from those proposed in the literature. Overall, the outcome was disappointing: no significant improvement in the quality of the estimates was provided by any of these joint models. For orthopaedic applications, estimates of knee joint kinematics should preferably be unbiased and it is desirable that they remain within a $2^{\circ}$ and 2 mm confidence interval (Iwaki et al., 2000). The MBO method and the six joint models evaluated in the present study did not reach this level of accuracy.

\subsection{Propagation of STA to joint kinematics}

Given the fact that the most satisfactory outcomes were provided by the MBO method embedding model None, it can be inferred that most of the skin-marker datasets analysed entailed limited STA. Still, virtual joint dislocation, common with this joint model, is revealed by the high $C I$ observed for joint displacements in most of the motor tasks. The results tend to support the conclusions presented in Andersen et al. (2010), in that the use of MBO embedding mechanical joint models (Spherical and Hinge) did not eliminate or significantly reduce the effects of STA and did not improve the overall validity of knee joint kinematics derived from skin-markers placed on the thigh and shank. The conclusion is the same for those models that are regarded as more physiological (Parallel, Coupling and Elastic), although performance did depend on the motor task and the DoF considered. The latter models were developed as a better option with respect to Spherical and Hinge models because 
they allow the estimate of joint displacements. The good to excellent determination coefficients obtained for anterior and proximal displacements support the notion that they can provide physiological time histories. However, from the results on bias $b, C I$ and $R M S E$, it is clear that joint displacement estimates remain inaccurate.

Most published evaluation studies could not count on the availability of artefact-free reference data and were based on skin-marker residuals (Andersen et al., 2009; Ojeda et al., 2014; Reinbolt et al., 2005) or joint dislocation in the case of model None (Lu and O'Connor, 1999; Ojeda et al., 2014; Sholukha et al., 2006). Only few studies provide a quantitative assessment of the effect of STA propagation to normal knee kinematics (i.e., non-pathological and nonoperated knees) using artefact-free reference data. For level walking and investigating six subjects with skin- and pin-mounted markers, Andersen et al. (2010) reported a mean RMSE between $1.4^{\circ}$ and $8.1^{\circ}$ for extension, $1.5^{\circ}$ and $9.7^{\circ}$ for adduction, and $1.3^{\circ}$ and $4.7^{\circ}$ for internal rotation when using model Spherical. For ten subjects, performing a squatting task with reference markers mounted on an external fixator and using biplane radiography, Clément et al. (2017) found higher RMSEs, with values up to $21.8^{\circ}$ for angles and up to 9.7 mm for displacements using knee models Spherical, Hinge, Coupling and Parallel. Mean RMSEs for angles and displacements, respectively, were in the ranges $\left[3.3^{\circ}-8.1^{\circ}\right]$ and $[1.8$ $\mathrm{mm}-2.9 \mathrm{~mm}]\left(\right.$ Spherical), $\left[4.3^{\circ}-9.2^{\circ}\right]$ and $[1.8 \mathrm{~mm}-2.9 \mathrm{~mm}]($ Hinge $),\left[4.5^{\circ}-13.2^{\circ}\right]$ and [1.8 mm - $5.5 \mathrm{~mm}]\left(\right.$ Coupling), and $\left[3.2^{\circ}-9.6^{\circ}\right]$ and $[1.6 \mathrm{~mm}-4.1 \mathrm{~mm}]\left(\right.$ Parallel), at $64^{\circ}$ of flexion. For running, with three subjects (skin- and pin-mounted markers), Gasparutto et al. (2015) found lower overall RMSEs with model Coupling for extension, adduction, and internal rotation (up to $2.0^{\circ}, 3.2^{\circ}, 5,5^{\circ}$, respectively) and for lateral, anterior and proximal displacements (up to $1.7 \mathrm{~mm} 4.5 \mathrm{~mm}$ and $2.5 \mathrm{~mm}$, respectively). In the same study by Gasparutto et al. (2015), the mean RMSEs were below $2.5^{\circ}$ and $4.1 \mathrm{~mm}$ and $2.2^{\circ}$ and $3.1 \mathrm{~mm}$ with model Parallel and Spherical, respectively, for joint angles and displacements. Model 
None provided the highest RMSE, with an overall mean of about $2.3^{\circ}$ and $3.6 \mathrm{~mm}$ for joint angles and displacements. Finally, for step-up with two subjects (skin markers and biplane fluoroscopy), Richard et al. (2016) reported RMSEs of up to $7.1^{\circ}$ and $8.2 \mathrm{~mm}$ for joint angles and displacements with model None, up to $5.6^{\circ}$ and $7 \mathrm{~mm}$ with model Spherical, up to $20.4^{\circ}$ and $3.5 \mathrm{~mm}$ with model Parallel, and up to $6.4^{\circ}$ and $3.6 \mathrm{~mm}$ with model Elastic. In the same study, the authors provide values for $b$ and $C I$ (maxima of the absolute values $[b ; C I]$ ) of up to $[1.9 ; 13.9]^{\circ}$ and $[4.2 ; 15.5] \mathrm{mm}$ for joint angles and displacements with model None, up to $[2.0 ; 11.0]^{\circ}$ and $[5.3 ; 9.2] \mathrm{mm}$ with model Spherical, up to $[19.4 ; 12.7]^{\circ}$ and $[3.4 ; 6.9] \mathrm{mm}$ with model Parallel and up to $[2.4 ; 12.3]^{\circ}$ and $[2.4 ; 7.0] \mathrm{mm}$ with model Elastic.

Overall, the values obtained for RMSE (or $b$ and $C I$ ) in the present study were lower than those reported in the literature for various motor tasks and with various means of assessing reference kinematics. This could be explained by the fact that the analysed datasets from skinmarkers involved small STAs, as demonstrated by the acceptable results obtained with model None. Assessing the degree of agreement through Bland-Altman (Bland and Altman, 1986) allowed a deeper comparative analysis of the joint models. For instance, Hinge and Coupling models, with numerous "hard" constraints, typically resulted in a higher bias $b$ for internal rotation. It was also expected that Parallel and Elastic models, which introduce "soft" constraints, would yield lower $C I$, but this did not occur systematically.

The contrasting results obtained using the same model for a given subject performing three different tasks show that a joint model may be better suited to one particular motor task, while other models might work better for other tasks. For instance, for hopping, model Coupling provided poor agreement on joint angles but good agreement on displacements, while model Elastic yielded opposite indications, with good agreement on angles and poor agreement on displacements. No doubt some assumptions on the dependent DoFs in the knee, as well as some physiological features (i.e., ligaments with minimal elongation, stiffness coefficients), 
all established from cadaveric measurements, are questionable when applied to certain motor tasks performed in vivo. In examining cutting and hopping, two motor tasks which have not been studied before, we selected a critical case to assess the joint models used in MBO. Knee kinematics during these tasks may actually be distinctive (i.e., large anterior and lateral displacements), due to muscle contractions as well as external loading. Moreover, the amplitude of STA may also be reduced due to the muscle contractions involved.

\subsection{Limitations}

This study presents a number of limitations. First, only one trial with one subject was studied for each motor task. Second, only two segments were considered in the MBO. Different results can be expected from using $\mathrm{MBO}$ that considers more segments and adding constraints for other joints (e.g., hip and ankle). As previously shown (Duprey et al., 2010), joint kinematics, when estimated using $\mathrm{MBO}$, depends on the type and the number of joint models considered. Third, our study considered pin-marker data as a reference (i.e. artefact-free), and the drawbacks of this approach are well known (Ramsey and Wretenberg, 1999). In particular, local anaesthesia of the soft tissues surrounding the zone where the pins were screwed into the bone may partially explain the small quantity of STA observed on the knee joint kinematics estimation provided by the MBO embedding model None. Fourth, modelling the lower limb with rigid segments and non-personalised joint constraints that impede or prescribe one or more DoFs may introduce errors. In that case the procedure, instead of compensating for STA as originally intended, adds modelling errors to it. Subject-specific joint models embedded in MBO, in particular models Spherical and Parallel (Clément et al., 2015), have been shown to have a beneficial impact on kinematics estimation.

\section{Conclusion}

The present study proposed a quantitative comparison of the estimation of knee joint kinematics using $\mathrm{MBO}$ embedding six different models versus reference (i.e., artefact-free) 
kinematics. As a conclusion, it came out that MBO cannot be considered a fully reliable solution to compensate for the STA. As a matter of fact, only invasive/ionising methods can currently give access to joint kinematics with the desirable level of accuracy ( $2^{\circ}$ and $2 \mathrm{~mm}$ ). Nevertheless MBO remains a useful approach for further biomechanical computation such as inverse dynamics and musculoskeletal modelling.

\section{Conflict of interest statement}

No party having a direct interest in the results of the research supporting this article has or will confer a benefit on the author(s) or on any organization with which the author(s) is/are associated.

\section{References}

Andersen, M.S., Benoit, D.L., Damsgaard, M., Ramsey, D.K., Rasmussen, J., 2010. Do kinematic models reduce the effects of soft tissue artefacts in skin marker-based motion analysis? An in vivo study of knee kinematics. J Biomech 43, 268-273.

Andersen, M.S., Damsgaard, M., Rasmussen, J., 2009. Kinematic analysis of overdeterminate biomechanical systems. Comput Methods Biomech Biomed Engin 12, 371-384.

Benoit, D.L., Ramsey, D.K., Lamontagne, M., Xu, L., Wretenberg, P., Renström, P., 2006. Effect of skin movement artifact on knee kinematics during gait and cutting motions measured in vivo. Gait Posture 24, 152-164.

Bland, J., Altman, D.G., 1986. Statistical Methods for Assessing Agreement Between Two Methods of Clinical Measurement. The Lancet 327, 307-310.

Bonnechère, B., Sholukha, V., Salvia, P., Rooze, M., Van Sint Jan, S., 2015. Physiologically corrected coupled motion during gait analysis using a model-based approach. Gait Posture 41, 319-322.

Cereatti, A., Bonci, T., Aminian, K., Barré, A., Begon, M., Benoit, D.L., Charbonnier, C., Lin, C.C., Dal Maso, F., Fantozzi, S., Lu, T.W., Stagni, R., Pandy, M.G., van den Bogert, A.J., Camomilla, V., 2016. A proposal for the standardization of soft tissue artefact description and data exchange, and an open dataset on upper and lower limb artefacts during different motor tasks. J Biomech, Submitted.

Charlton, I.W., Tate, P., Smyth, P., Roren, L., 2004. Repeatability of an optimised lower body model. Gait Posture 20, 213-221.

Chiari, L., Croce, U.D., Leardini, A., Cappozzo, A., 2005. Human movement analysis using stereophotogrammetry: Part 2: Instrumental errors. Gait Posture 21, 197-211. 
Clément, J., Dumas, R., Hagemeister, N., de Guise, J.A., 2015. Soft tissue artifact compensation in knee kinematics by multi-body optimization: Performance of subjectspecific knee joint models. J Biomech 48, 3796-3802.

Clément, J., Dumas, R., Hagemeister, N., de Guise, J.A., 2017. Can generic knee joint models improve the measurement of osteoarthritic knee kinematics during squatting activity? Computer Methods in Biomechanics and Biomedical Engineering 20, 94-103.

de Jalon, J.G., Cuadrado, J., Avello, A., Jimenez, J.M., 1994. Kinematic and Dynamic Simulation of Rigid and Flexible Systems with Fully Cartesian Coordinates, in: Seabra Pereira, M.F.O., Ambrósio, J.A.C. (Eds.), Computer-Aided Analysis of Rigid and Flexible Mechanical Systems. Springer Netherlands, Dordrecht, pp. 285-323.

Dumas, R., Chèze, L., 2007. 3D inverse dynamics in non-orthonormal segment coordinate system. Medical \& Biological Engineering \& Computing 45, 315-322.

Dumas, R., Robert, T., Pomero, V., Cheze, L., 2012. Joint and segment coordinate systems revisited. Computer Methods in Biomechanics and Biomedical Engineering 15, 183-185.

Duprey, S., Cheze, L., Dumas, R., 2010. Influence of joint constraints on lower limb kinematics estimation from skin markers using global optimization. J Biomech 43, 28582862.

Gasparutto, X., Sancisi, N., Jacquelin, E., Parenti-Castelli, V., Dumas, R., 2015. Validation of a multi-body optimization with knee kinematic models including ligament constraints. J Biomech 48, 1141-1146.

Iwaki, H., Pinskerova, V., Freeman, M. A. R., 2000. Tibiofemoral movement 1: the shapes and relative movements of the femur and tibia in the unloaded cadaver knee. $\mathrm{J}$ Bone Joint Surg [Br] 82-B, 1189-1195.

Leardini, A., Chiari, L., Croce, U.D., Cappozzo, A., 2005. Human movement analysis using stereophotogrammetry: Part 3. Soft tissue artifact assessment and compensation. Gait Posture 21, 212-225.

Li, K., Zheng, L., Tashman, S., Zhang, X., 2012. The inaccuracy of surface-measured modelderived tibiofemoral kinematics. J Biomech 45, 2719-2723.

Lu, T.W., O'Connor, J.J., 1999. Bone position estimation from skin marker co-ordinates using global optimisation with joint constraints. J Biomech 32, 129-134.

McLaughlin, P., 2013. Testing agreement between a new method and the gold standardHow do we test? J Biomech 46, 2757-2760.

Ojeda, J., Martínez-Reina, J., Mayo, J., 2014. A method to evaluate human skeletal models using marker residuals and global optimization. Mechanism and Machine Theory 73, 259272.

Parenti-Castelli, V., Sancisi, N., 2013. Synthesis of Spatial Mechanisms to Model Human Joints, in: McCarthy, J.M. (Ed.), 21st Century Kinematics: The 2012 NSF Workshop. Springer London, London, pp. 49-84.

Peters, A., Galna, B., Sangeux, M., Morris, M., Baker, R., 2010. Quantification of soft tissue artifact in lower limb human motion analysis: A systematic review. Gait Posture 31, 1-8.

Ramsey, D.K., Wretenberg, P.F., 1999. Biomechanics of the knee: methodological considerations in the in vivo kinematic analysis of the tibiofemoral and patellofemoral joint. Clinical Biomechanics 14, 595-611.

Reinbolt, J.A., Schutte, J.F., Fregly, B.J., Koh, B.I., Haftka, R.T., George, A.D., Mitchell, K.H., 2005. Determination of patient-specific multi-joint kinematic models through two-level optimization. J Biomech 38, 621-626.

Reinschmidt, C., van den Bogert, A.J., Nigg, B.M., Lundberg, A., Murphy, N., 1997. Effect of skin movement on the analysis of skeletal knee joint motion during running. J Biomech 30, 729-732. 
Richard, V., Lamberto, G., Lu, T.-W., Cappozzo, A., Dumas, R., 2016. Knee Kinematics Estimation Using Multi-Body Optimisation Embedding a Knee Joint Stiffness Matrix: A Feasibility Study. PLoS ONE 11, e0157010.

Scheys, L., Desloovere, K., Spaepen, A., Suetens, P., Jonkers, I., 2011. Calculating gait kinematics using MR-based kinematic models. Gait Posture 33, 158-164.

Sholukha, V., Leardini, A., Salvia, P., Rooze, M., Van Sint Jan, S., 2006. Double-step registration of in vivo stereophotogrammetry with both in vitro 6-DOFs electrogoniometry and CT medical imaging. J Biomech 39, 2087-2095.

Soderkvist, I., Wedin, P.A., 1993. Determining the movements of the skeleton using wellconfigured markers. J Biomech 26, 1473-1477.

Tsai, T.-Y., Lu, T.-W., Kuo, M.-Y., Lin, C.-C., 2011. Effects of soft tissue artifacts on the calculated kinematics and kinetics of the knee during stair-ascent. Journal of Biomechanics 44, 1182-1188.

Valente, G., Pitto, L., Stagni, R., Taddei, F., 2015. Effect of lower-limb joint models on subject-specific musculoskeletal models and simulations of daily motor activities. J Biomech 48, 4198-4205.

Walker, P.S., Rovick, J.S., Robertson, D.D., 1988. The effects of knee brace hinge design and placement on joint mechanics. J Biomech 21, 965-967.

Wu, G., Siegler, S., Allard, P., Kirtley, C., Leardini, A., Rosenbaum, D., Whittle, M., D'Lima, D.D., Cristofolini, L., Witte, H., Schmid, O., Stokes, I., 2002. ISB recommendation on definitions of joint coordinate system of various joints for the reporting of human joint motion--part I: ankle, hip, and spine. International Society of Biomechanics. J Biomech 35, 543-548. 


\section{Figure and table captions}

Figure 1. Level walking. Knee joint angles and displacements estimated using MBO method embedding each of the six knee joint models: no joint model (None, red), spherical model (Spherical, blue), hinge model (Hinge, cyan), parallel mechanism (Parallel, orange), coupling curves model (Coupling, purple), and stiffness matrix (Elastic, green) plotted against reference kinematics, i.e., pin-marker data (Reference, black).

Figure 2. Cutting. Knee joint angles and displacements estimated using MBO method embedding each of the six knee joint models: no joint model (None, red), spherical model (Spherical, blue), hinge model (Hinge, cyan), parallel mechanism (Parallel, orange), coupling curves model (Coupling, purple), and stiffness matrix (Elastic, green) plotted against reference kinematics, i.e., pin-marker data (Reference, black).

Figure 3. Hopping. Knee joint angles and displacements estimated using MBO method embedding each of the six knee joint models: no joint model (None, red), spherical model (Spherical, blue), hinge model (Hinge, cyan), parallel mechanism (Parallel, orange), coupling curves model (Coupling, purple), and stiffness matrix (Elastic, green) plotted against reference kinematics, i.e., pin-marker data (Reference, black).

Table 1. Level walking. Bland Altman bias $(b)$ and confidence interval $(C I)$ as well as correlation coefficient $\left(R^{2}\right)$ and root mean square error (RMSE) when comparing results of the MBO method embedding the six different knee joint models None, Spherical, Hinge, Parallel, Coupling and Elastic considering the extension, adduction and internal rotation angles as well as the lateral, anterior and proximal displacements versus the reference. Except for $R^{2}$ (no unit), the units are degrees for angles and $\mathrm{mm}$ for displacements.

Table 2. Cutting. Bland Altman bias $(b)$ and confidence interval $(C I)$ as well as correlation coefficient $\left(R^{2}\right)$ and root mean square error (RMSE) when comparing results of the MBO 
method embedding the six different knee joint models None, Spherical, Hinge, Parallel, Coupling and Elastic considering the extension, adduction and internal rotation angles as well as the lateral, anterior and proximal displacements versus the reference. Except for $R^{2}$ (no unit), the units are degrees for angles and $\mathrm{mm}$ for displacements.

Table 3. Hopping. Bland Altman bias $(b)$ and confidence interval $(C I)$ as well as correlation coefficient $\left(R^{2}\right)$ and root mean square error (RMSE) when comparing results of the MBO method embedding the six different knee joint models None, Spherical, Hinge, Parallel, Coupling and Elastic considering the extension, adduction and internal rotation angles as well as the lateral, anterior and proximal displacements versus the reference. Except for $R^{2}$ (no unit), the units are degrees for angles and $\mathrm{mm}$ for displacements. 

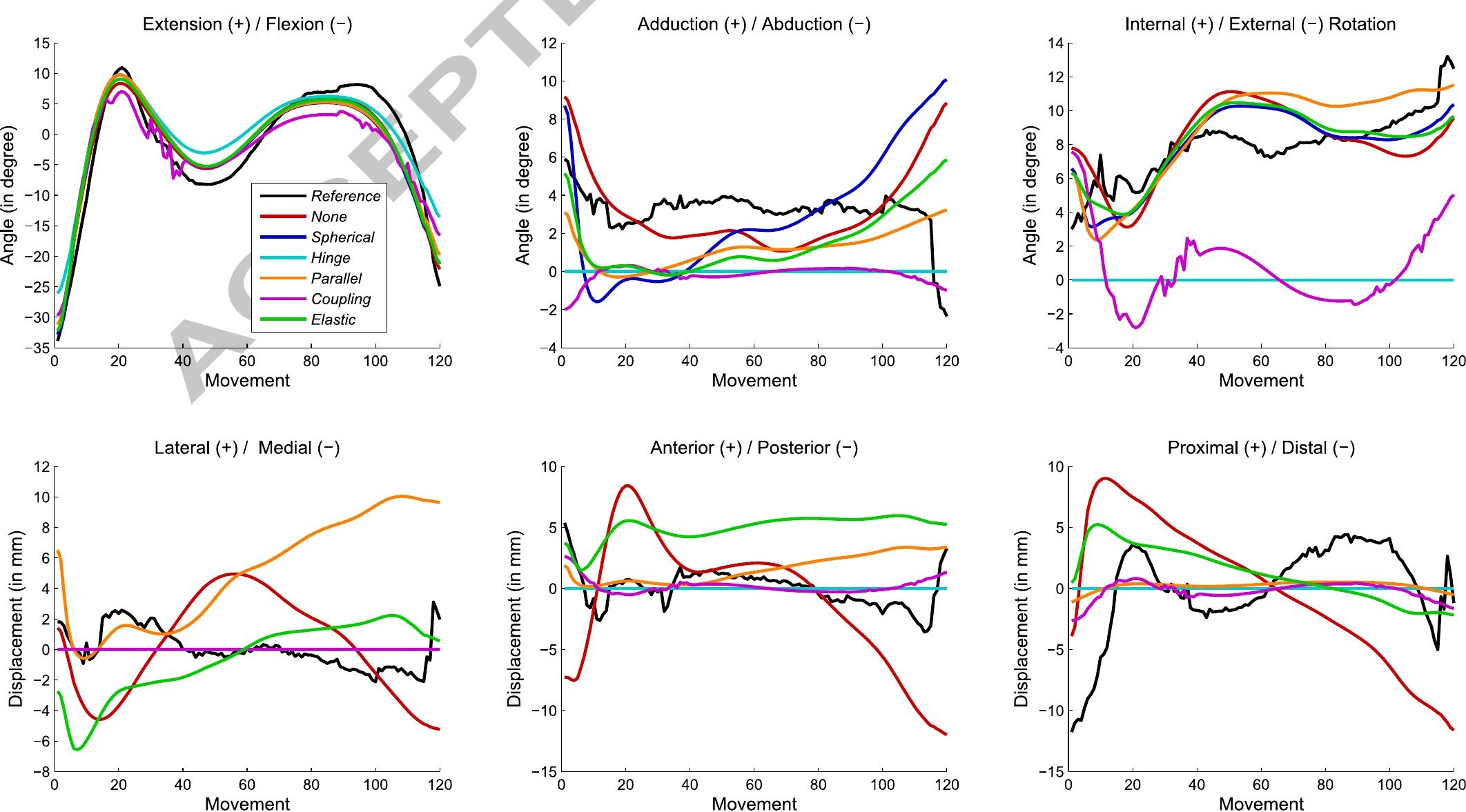
Figure 2
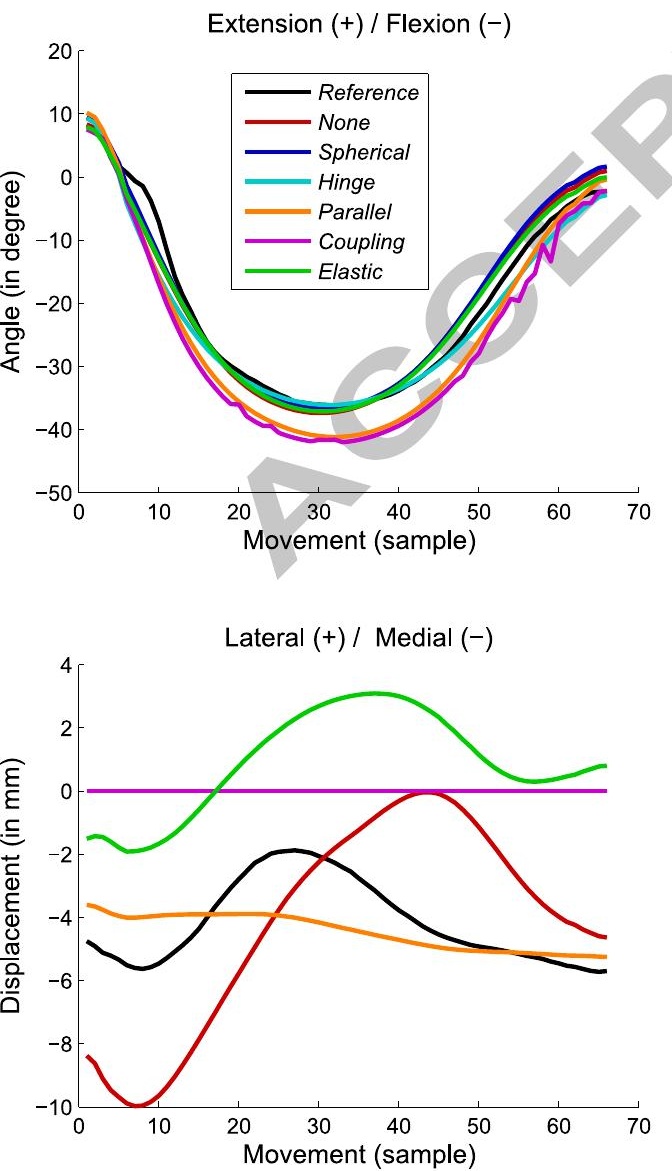

Adduction (+) / Abduction (-)

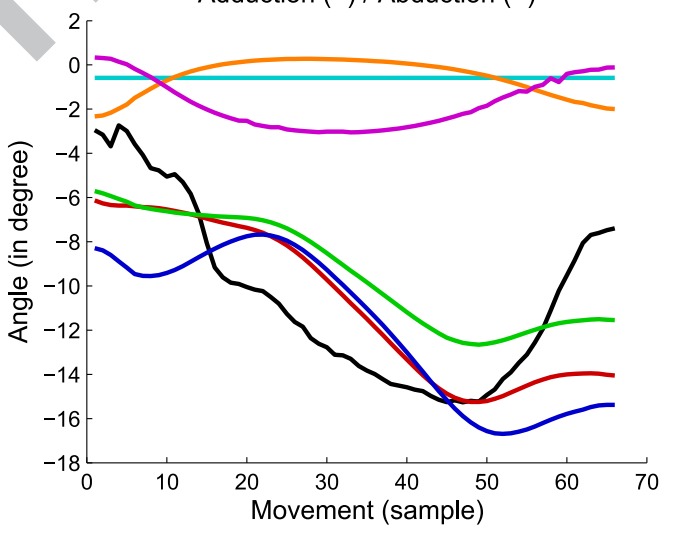

Anterior (+) / Posterior (-)

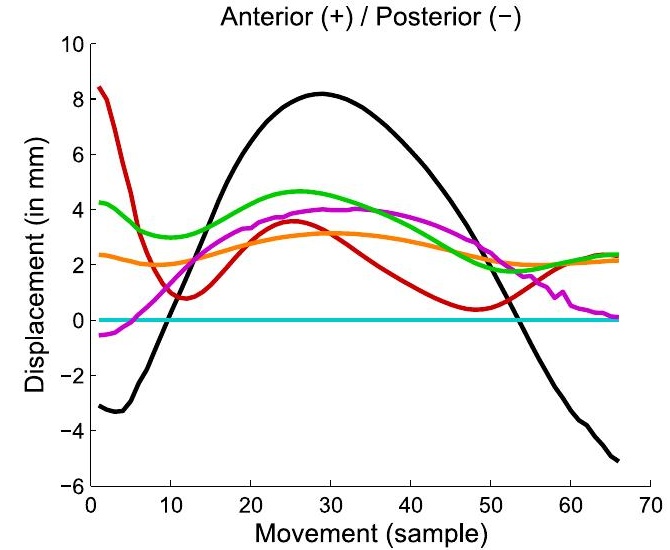

Internal (+) / External (-) Rotation

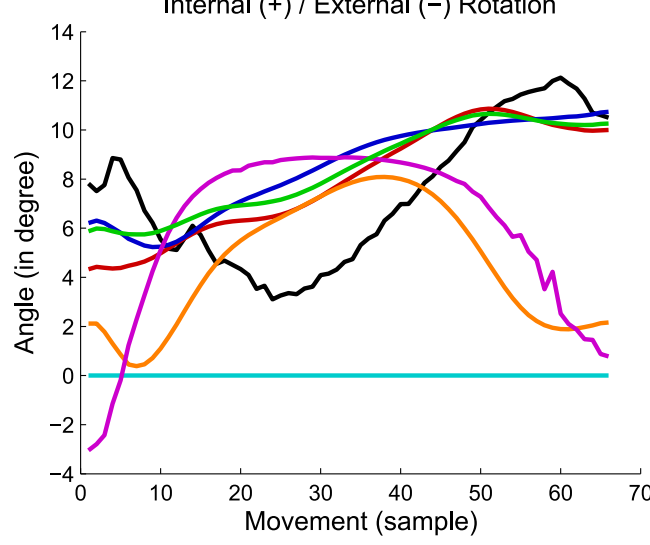

Proximal (+) / Distal (-)

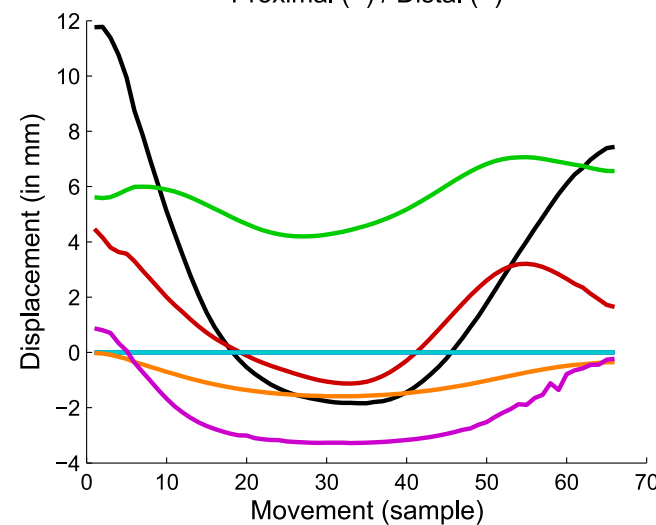


Figure 3
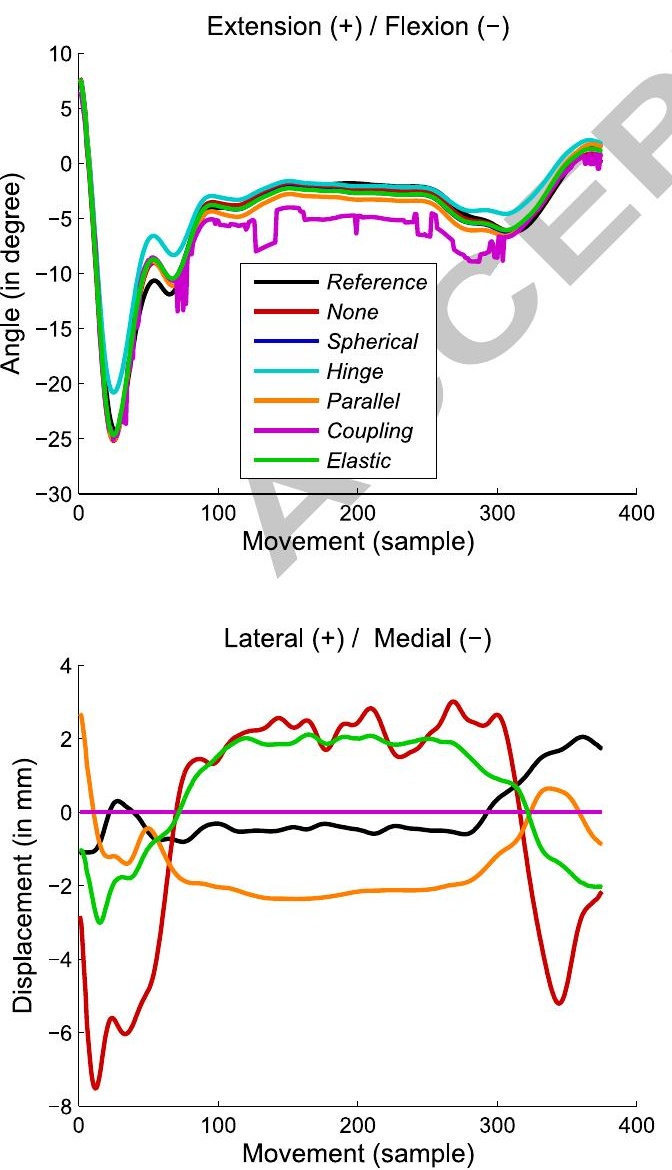

Adduction (+) / Abduction (-)
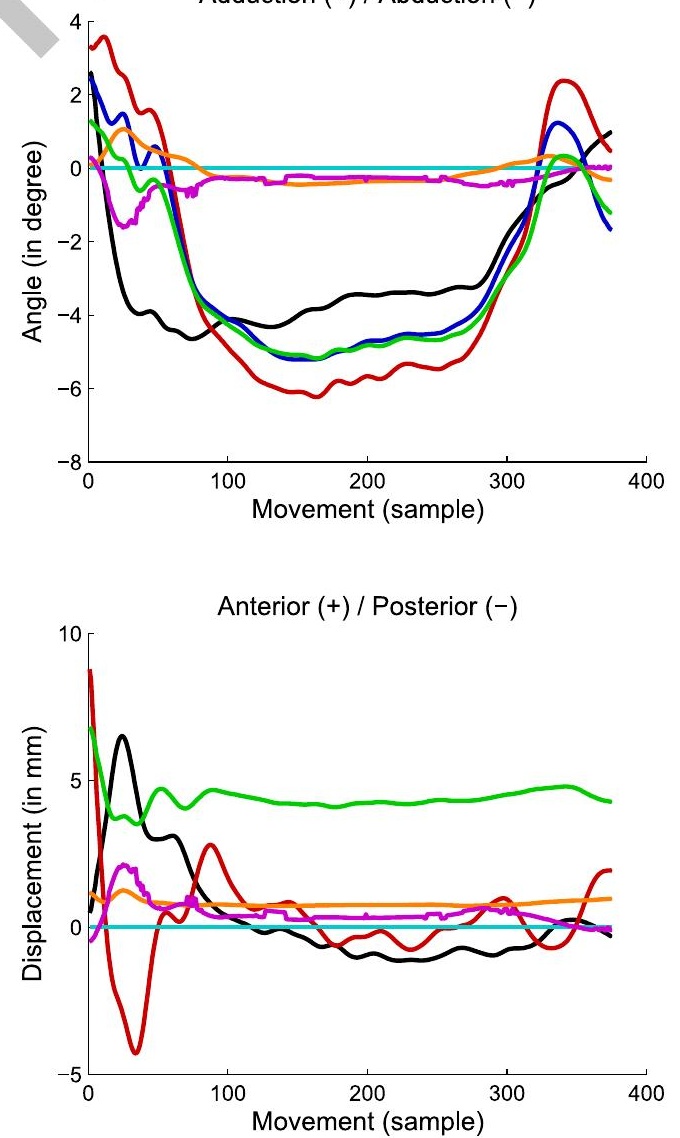
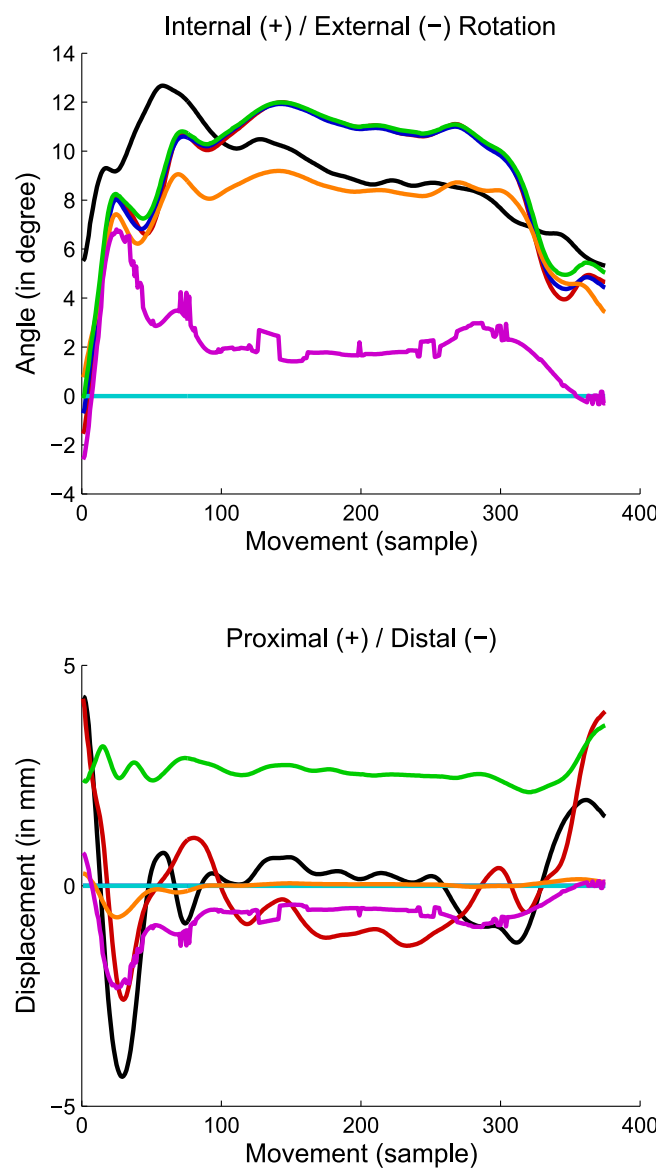
Table 1

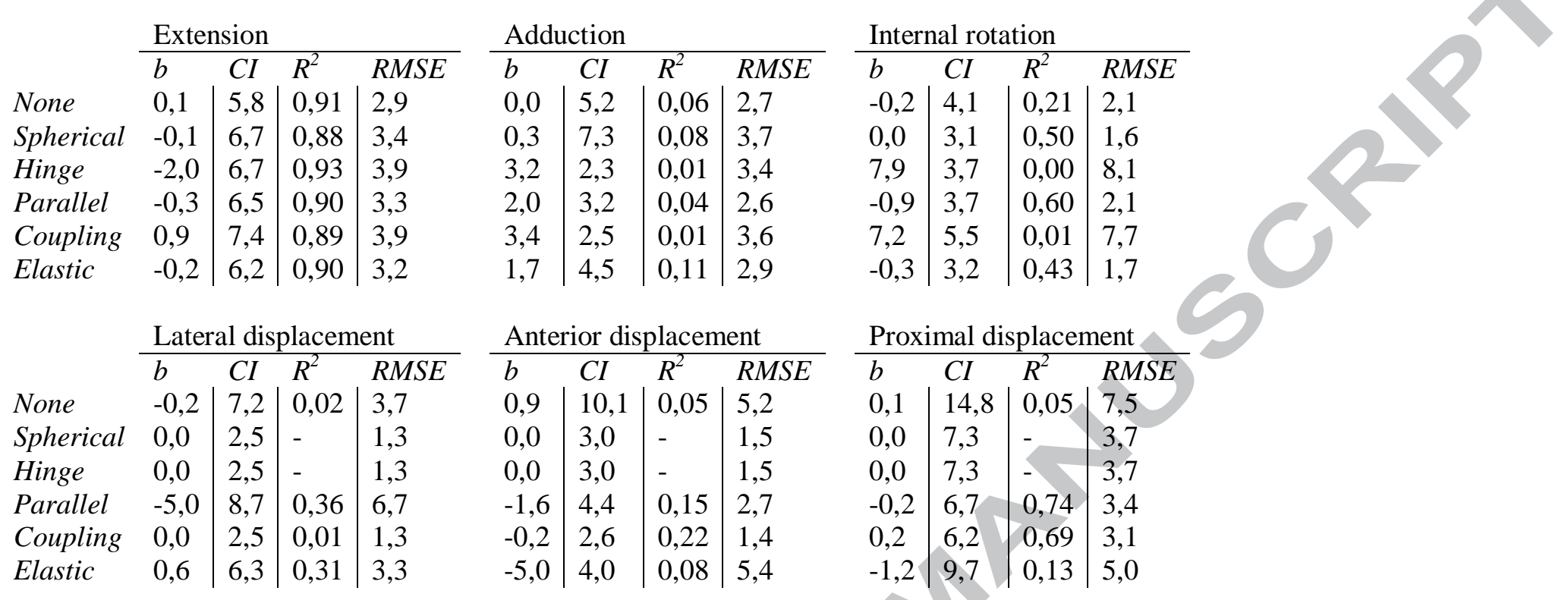


Table 2

\begin{tabular}{ll|l|l|l} 
& \multicolumn{4}{l}{ Extension } \\
\cline { 2 - 5 } None & $b$ & \multicolumn{1}{l}{$C I$} & $R^{2}$ & $R M S E$ \\
Spherical & $-0,2$ & 5,1 & 0,97 & 2,6 \\
Hinge & 1,5 & 4,8 & 0,97 & 2,4 \\
Parallel & 3,6 & 3,8 & 0,98 & 2,4 \\
Coupling & 4,4 & 4,9 & 0,98 & 4,3 \\
Elastic & 0,1 & 4,5 & 0,99 & 5,0 \\
& \multicolumn{4}{|l}{} \\
& Lateral displacement \\
\cline { 2 - 5 } & $b$ & $C I$ & $R^{2}$ & $R M S E$ \\
None & $-0,4$ & 6,2 & 0,27 & 3,2 \\
Spherical & 0,2 & 2,9 & - & 1,5 \\
Hinge & 0,2 & 2,9 & - & 1,5 \\
Parallel & 4,2 & 3,1 & 0,00 & 4,5 \\
Coupling & 0,2 & 2,9 & 0,01 & 1,5 \\
Elastic & $-2,1$ & 2,3 & 0,40 & 2,4
\end{tabular}

\begin{tabular}{|c|c|c|c|}
\hline \multicolumn{4}{|c|}{ Adduction } \\
\hline$b$ & $C I$ & $R^{2}$ & $R M S E$ \\
\hline 0,3 & 5,5 & 0,45 & 2,8 \\
\hline 0,4 & 6,0 & 0,33 & 3,1 \\
\hline$-10,2$ & 6,6 & 0,02 & 10,7 \\
\hline$-10,2$ & 7,6 & 0,41 & 10,9 \\
\hline$-8,4$ & 5,1 & 0,56 & 8,8 \\
\hline$-1,0$ & 4,7 & 0,50 & 2,5 \\
\hline
\end{tabular}

Internal rotation

b $\quad C I \quad R^{2} \quad$ RMSE

\begin{tabular}{l|l|l|l}
$-0,6$ & 4,4 & 0,41 & 2,3
\end{tabular}

\begin{tabular}{l|l|l|l}
$-0,5$ & 4,4 & 0,38 & 2,3
\end{tabular}

\begin{tabular}{l|l|l|l}
$-0,5$ & 4,4 & 0,38 & 2,3 \\
7,3 & 5,6 & 0,01 & 7,8
\end{tabular}

\begin{tabular}{l|l|l|l|l}
2,4 & 8,1 & 0,13 & 4,8
\end{tabular}

\begin{tabular}{l|l|l|l}
1,5 & 11,1 & 0,32 & 5,8
\end{tabular}

\begin{tabular}{l|l|l|l}
1,5 & 11,1 & 0,32 & 5,8 \\
$-0,6$ & 4,0 & 0,52 & 2,1
\end{tabular}

Anterior displacement

\begin{tabular}{llll}
\hline$b$ & $C I$ & $R^{2}$ & RMSE
\end{tabular}

Proximal displacement

\begin{tabular}{l|l|l|lll|l|l|l}
\hline$b$ & $C I$ & $R^{2}$ & RMSE & & \multicolumn{3}{l}{$b$} & \multicolumn{2}{l}{$C I$} & $R^{2}$ & $R M S E$ \\
1,3 & 11,8 & 0,31 & 6,1 & & 0,9 & 7,5 & 0,31 & 3,9 \\
0,0 & 9,5 & - & 4,8 & & 0,1 & 8,7 & - & 4,4 \\
0,0 & 9,5 & - & 4,8 & & 0,1 & 8,7 & - & 4,4 \\
$-2,4$ & 8,6 & 0,78 & 5,0 & & 1,0 & 7,7 & 0,93 & 4,0 \\
$-2,3$ & 6,5 & 0,96 & 4,0 & & 2,2 & 6,3 & 0,95 & 3,9 \\
$-3,1$ & 9,7 & 0,00 & 5,8 & & $-4,5$ & 8,6 & 0,03 & 6,3
\end{tabular}


Table 3

\begin{tabular}{ll|l|l|l} 
& \multicolumn{4}{l}{ Extension } \\
\cline { 2 - 5 } None & $b$ & $C I$ & $R^{2}$ & $R M S E$ \\
Spherical & 0,0 & 1,3 & 0,98 & 0,7 \\
Hinge & $-1,1$ & 2,6 & 0,98 & 0,8 \\
Parallel & 0,4 & 1,9 & 0,96 & 1,7 \\
Coupling & 1,7 & 3,6 & 0,89 & 1,1 \\
Elastic & 0,0 & 1,6 & 0,98 & 0,8 \\
& \multicolumn{4}{l}{} \\
& \multicolumn{5}{l}{ Lateral displacement } \\
\cline { 2 - 5 } None & 0,0 & 7,0 & 0,16 & 3,6 \\
Spherical & $-0,1$ & 1,6 & - & 0,8 \\
Hinge & $-0,1$ & 1,6 & - & 0,8 \\
Parallel & 1,4 & 1,7 & 0,33 & 1,6 \\
Coupling & $-0,1$ & 1,6 & 0,00 & 0,8 \\
Elastic & $-0,7$ & 4,1 & 0,26 & 2,2
\end{tabular}

\begin{tabular}{l|l|l|l}
\multicolumn{4}{l}{ Adduction } \\
\hline$b$ & $C I$ & $R^{2}$ & $R M S E$ \\
$-0,1$ & 5,1 & 0,42 & 2,6 \\
$-0,2$ & 3,6 & 0,40 & 1,9 \\
$-2,9$ & 3,3 & 0,00 & 3,3 \\
$-2,8$ & 3,2 & 0,04 & 3,2 \\
$-2,5$ & 3,1 & 0,16 & 2,9 \\
0,1 & 3,1 & 0,44 & 1,6
\end{tabular}

Internal rotation

$\begin{array}{llll}b & C I & R^{2} & R M S E\end{array}$

\begin{tabular}{l|l|l|l}
$-0,1$ & 4,8 & 0,26 & 2,5
\end{tabular}

\begin{tabular}{lll|l|l}
$-0,2$ & 4,6 & 0,28 & 2,3
\end{tabular}

\begin{tabular}{lll|l|l}
9,0 & 3,5 & 0,00 & 9,2
\end{tabular}

\begin{tabular}{lll|l|l}
1,5 & 3,1 & 0,35 & 2,2
\end{tabular}

\begin{tabular}{l|l|l|l|l}
6,8 & 3,1 & 0,29 & 7,0
\end{tabular}

\begin{tabular}{l|l|l|l}
$-0,4$ & 4,3 & 0,28 & 2,2
\end{tabular}

Anterior displacement

Proximal displacement \begin{tabular}{llll}
\hline$b$ & $C I$ & $R^{2}$ & $R M S E$
\end{tabular} \begin{tabular}{l|l|l|l}
0,2 & 5,4 & 0,10 & 2,8
\end{tabular} \begin{tabular}{l|l|l|l|l}
0,4 & 3,6 & - & 1,9
\end{tabular}

$\begin{array}{lllll}0,4 & 3,6 & - & 1,9\end{array}$

\begin{tabular}{l|l|l|l|l}
$-0,4$ & 3,4 & 0,48 & 1,8
\end{tabular}

$\begin{array}{llllll}-0,1 & 3,0 & 0,56 & 1,5\end{array}$

\begin{tabular}{l|l|l|l}
$-4,0$ & 3,8 & 0,05 & 4,4
\end{tabular}

\begin{tabular}{llll}
\hline$b$ & $C I$ & $R^{2}$ & $R M S E$
\end{tabular}

\begin{tabular}{l|l|l|l}
0,1 & 2,3 & 0,37 & 1,2
\end{tabular}

\begin{tabular}{ll|l|l}
0,0 & 2,5 & - & 1,3
\end{tabular}

$\begin{array}{llll}0,0 & 2,5 & - & 1,3\end{array}$

\begin{tabular}{ll|l|l|l}
0,0 & 2,3 & 0,70 & 1,2
\end{tabular}

\begin{tabular}{l|l|l|l}
0,7 & 1,7 & 0,82 & 1,1
\end{tabular}

\begin{tabular}{l|l|l|l}
$-2,6$ & 2,5 & 0,05 & 2,9
\end{tabular} 